\title{
Nutrição Esportiva como Componente Curricular nos Cursos de Nutrição e Educação Física do Centro-Oeste do Brasil
}

\section{Sports Nutrition as Curricular Component in the Nutrition and Physical Education Courses of the Center-West of Brazil}

\author{
Adélia Mascarenhas de Sousa Lima ${ }^{1}$ \\ Tatiana Evangelista da Silva Rocha ${ }^{2}$ \\ Tatienne Neder Figueira da Costa ${ }^{2}$
}

\section{RESUMO}

Objetivo: Esse estudo objetivou caracterizar a disciplina de Nutrição Esportiva nos cursos de Graduação em Nutrição e Educação Física das Instituições de Ensino Superior do Centro-Oeste do Brasil e Distrito Federal. Material e Métodos: Adotou-se como critério de inclusão os cursos presenciais ou de educação à distância de Graduação em Nutrição (Bacharelado) e Educação Física (Bacharelado e Licenciatura) das Instituições de Ensino Superior reconhecidas pelo Ministério da Educação. Foram analisados os três estados que compõem a Região Centro-Oeste do Brasil e o Distrito Federal. As Instituições foram selecionadas por meio da Base de Dados do Sistema de Regulação do Ensino Superior - Sistema e-MEC e para a avaliação considerou-se a presença da disciplina Nutrição Esportiva e sua caracterização de oferta como sua denominação; natureza (obrigatória ou optativa); carga horária e período ministrado. Resultados: Observou-se que para $100 \%$ dos cursos de Nutrição a disciplina é de natureza obrigatória, ao passo para que para a Educação Física esse percentual foi de 95,23\%. Ambos os cursos apresentaram ampla variabilidade da carga horária total e maior carga horária teórica, quando comparada à carga horária prática. Também, alguns estados não destinam nenhuma carga horária prática para sua realização. Conclusão: Apesar de os cursos apresentarem similaridade em seu perfil de oferta para a maioria dos aspectos avaliados, faz-se necessário entender se as diferenças encontradas impactam no processo de ensino-aprendizagem da disciplina estudada.

DESCRITORES: Currículo. Ensino. Nutrição. Exercício.

\begin{abstract}
Objective: This study aimed to characterize the discipline of Sports Nutrition in the Nutrition and Physical Education Undergraduate courses of the Institutions of Higher Education of the Center-West of Brazil and the Federal District. Material and Methods: The adopted inclusion criteria were the presential or distance education courses of Undergraduate Nutrition (Bachelor) and Physical Education (Bachelor and Graduation) of Higher Education Institutions recognized by the Ministry of Education. The three states that compose the Central-West Region of Brazil and the Federal District were analyzed. The Institutions were selected through the Database of the System of Regulation of Higher Education - e-MEC System and for the evaluation, it was considered the presence of the discipline of Sports Nutrition and its offer characterization as its denomination; nature (required or optional); workload and time period. Results: It was observed that for $100 \%$ of the Nutrition courses the discipline is required, whereas for Physical Education this percentage was $95.23 \%$. Both courses presented wide variability of the total workload and higher theoretical workload compared to the practical workload. Also, some states do not allocate any practical workload for their realization. Conclusion: Although the courses present similarity in their offer profile for most aspects evaluated, it is necessary to understand if the differences found, impact on the teaching-learning process of the studied discipline.
\end{abstract}

DESCRIPTORS: Curriculum. Teaching. Nutrition. Exercise.

\footnotetext{
1- Nutricionista Educacional na Diretoria Regional de Educação de Araguaína e Região e Nutricionista Ambulatorial na Clínica e Laboratório Biovida, Araguaína, TO, Brasil

2- Docente, Curso de Graduação em Nutrição, Universidade Federal do Tocantins, UFT, Palmas, TO, Brasil
} 
$\mathrm{O}$ exercício físico representa uma condição que impõe alterações nas necessidades nutricionais do indivíduo, por induzir adaptações metabólicas no organismo ${ }^{1}$. Pesquisas na área de nutrição e exercício têm crescido substancialmente nas últimas décadas, fator que tem contribuído para o reconhecido papel da nutrição na maximização da performance física.

Por outro lado, muitas vezes os atletas são dotados de crenças alimentares sem fundamentação, além de serem expostos a informações nutricionais de múltiplas fontes. Um estudo demonstrou que as fontes primárias de nutrição utilizadas pelos atletas advêm dos especialistas em condicionamento e força $(16,2 \%)$, treinadores $(11,4 \%)$ e técnicos $(7,7 \%)^{2}$, similar a estudos prévios $^{3}$. Tal comportamento pode ser explicado pela relação diária estabelecida com esses profissionais, bem como pela responsabilidade que os mesmos exercem para o bem estar geral dos atletas. Isso implica que conteúdos relacionados à nutrição esportiva não sejam restritos apenas aos cursos de graduação em nutrição, mas também na formação dos profissionais de educação física.

Os cursos de Nutrição e Educação Física são considerados da área da saúde e apresentam em suas Diretrizes Curriculares Nacionais (DCNs) um perfil profissional semelhante, que deve ser generalista, humanista e crítico ${ }^{4,5}$.

Além disso, as DCNs descrevem as competências que cada profissional deve desenvolver. Observa-se que os dois profissionais possuem semelhanças em sua formação, pois devem desenvolver habilidades de promoção da saúde.

Cabe ao profissional nutricionista atuar, visando à segurança alimentar e a atenção dietética, em todas as áreas do conhecimento em que alimentação e nutrição se apresentem fundamentais para a promoção, manutenção e a recuperação da saúde, bem como para a prevenção de doenças de indivíduos ou grupos populacionais, contribuindo para a melhoria da qualidade de vida ${ }^{4}$.

O profissional de educação física deve ser qualificado para analisar criticamente a realidade social, para nela intervir acadêmica e profissionalmente, por meio das diferentes manifestações e expressões do movimento humano, nas perspectivas da prevenção de problemas de agravo da saúde, promoção, proteção e reabilitação da saúde, visando a formação, a ampliação e o enriquecimento cultural das pessoas, para aumentar as possibilidades de adoção de um estilo de vida fisicamente ativo e saudável ${ }^{5}$.

A Resolução atual do Conselho Federal de Nutricionistas (CFN), $n^{\circ} .600$ (até então, a vigente era a $n^{\circ} .380$ ), desde 2005, reconhece a área de Nutrição em Esportes como uma das áreas de atuação desse profissional ${ }^{6}$. Em contrapartida, a Diretriz do curso de Graduação em Educação Física não é clara quanto à exigência de disciplinas que abordem conteúdos relacionados à nutrição. Contudo, na DCN deste curso é descrito que a Instituição de Ensino Superior (IES) poderá incorporar outras competências e habilidades que se mostrem adequadas e coerentes com seus projetos pedagógicos e também que a formação do graduado em Educação Física deve assegurar a indissociabilidade entre teoria e prática, por meio da prática como componente curricular ${ }^{5}$.

Proporcional à crescente procura pelo esporte e alimentação saudável entre atletas e indivíduos fisicamente ativos, aumenta-se também a demanda por profissionais capacitados. Segundo dados da International Health, Racquet \& Sportsclub Association (2016), o Brasil ocupa o segundo lugar no ranking mundial em número de academias (31.809), perdendo apenas para os Estados Unidos, com 36.180 academias $^{7}$. Em decorrência desse campo de atuação profissional cada vez mais promissor, faz-se necessária a inserção obrigatória da disciplina de Nutrição no Esporte na matriz curricular dos cursos de Graduação em Nutrição e Educação Física nas IES. Por esta razão, o objetivo do presente estudo foi avaliar e caracterizar a presença ou ausência da disciplina de Nutrição no Esporte nos cursos de Graduação em Nutrição e Educação Física da Região Centro-Oeste do Brasil.

\section{METODOLOGIA}

Trata-se de um estudo transversal e descritivo, 
realizado entre os meses de março e setembro do ano de 2016. Adotou-se como critério de inclusão os cursos presenciais ou de educação à distância de Graduação em Nutrição (Bacharelado) e Educação Física (Bacharelado e Licenciatura) das IES reconhecidas pelo Ministério da Educação (MEC). Foram analisados os três estados que compõem a Região Centro-Oeste do Brasil, sendo eles: Goiás (GO), Mato Grosso (MT) e Mato Grosso do Sul (MS), além do Distrito Federal (DF). As Instituições foram selecionadas por meio da Base de Dados do Sistema de Regulação do Ensino Superior - Sistema e-MEC do Ministério da Educação, disponível na Rede Mundial de Computadores.

Considerou-se IES: Universidades, Centros Universitários e Faculdades, Estatais e Particulares. Das IES registradas, foram excluídas as que se encontravam em extinção pelo sistema e-MEC ou que não fosse localizado o curso no site da referida Instituição. Para as IES inclusas em um primeiro momento, realizou-se a busca pelo Projeto Pedagógico de Curso (PPC), disponível nos sítios eletrônicos oficiais das IES. Na ausência do PPC, adotou-se como critério a consulta da matriz curricular atualizada de cada curso. Como último recurso, os pesquisadores contataram, via e-mail e/ou telefone, a coordenação do curso em questão para a obtenção dos dados necessários. Caso os recursos supracitados não fossem atendidos, o curso seria excluído do estudo.

Em um segundo momento, excluiu-se da pesquisa todas as IES/cursos que não obtinham a disciplina de Nutrição em Esportes em sua matriz curricular ou não fornecessem todas as informações referentes à caracterização da mesma: sua denominação; natureza (obrigatória ou optativa); carga horária (total, teórica e prática) e período ministrado. As variáveis foram analisadas em nível regional e interestadual, separadas por curso (Nutrição e Educação Física).

Para o tratamento estatístico, realizado pelo programa Microsoft Office Excel 2010, utilizou-se a frequência absoluta e relativa, moda, amplitude, médias e desvio-padrão.

\section{RESULTADOS}

Segundo dados do sistema e-MEC, na Região Centro-Oeste do Brasil e DF existem 33 cursos de Graduação em Nutrição cadastrados. No entanto, dois se encontram em extinção e quatro foram excluídos por não atenderem os critérios de busca pré-estabelecidos. Dos 27 cursos remanescentes, seis não ofertam a disciplina avaliada no presente estudo. Desse modo, foram analisados 21 cursos de graduação em Nutrição (Tabela 1), sendo $19,0 \%$ ofertados por IES públicas e $80,9 \%$ por instituições privadas.

Em relação aos cursos de Educação Física foram encontrados 117 cursos cadastrados no sistema e-MEC. Entretanto, três estavam em extinção e quatro não atenderam aos critérios de busca pré-estabelecidos. Dos 110 cursos restantes, 47 não ofertam a disciplina de nutrição em esportes e, portanto, 63 cursos entraram para a análise do presente estudo, sendo $44,4 \%$ na forma de licenciatura e 55,6\% como bacharelado (Tabela 2).

Tabela 1. Distribuição dos cursos de Graduação em Nutrição por Estado e DF, na Região Centro-Oeste do Brasil.

\begin{tabular}{l|c|c}
\multirow{2}{*}{ Estado e DF* } & \multicolumn{2}{|c}{ Cursos } \\
\cline { 2 - 3 } & $\mathbf{n = 2 1}$ & $\%$ \\
\hline Goiás & 7 & 33,3 \\
Distrito Federal & 7 & 33,3 \\
Mato Grosso & 3 & 14,3 \\
Mato Grosso do Sul & 4 & 19,0 \\
\hline \multicolumn{1}{c}{ Total } & 21 & $100 \%$ \\
\hline \multicolumn{2}{c}{}
\end{tabular}


Tabela 2. Distribuição dos cursos de Graduação em Educação Física (Licenciatura e Bacharelado) por Estado e DF, na Região Centro-Oeste do Brasil.

\begin{tabular}{l|c|c|c|c|c}
\hline \multirow{2}{*}{ Estado e DF* } & \multicolumn{2}{|c|}{ Licenciatura } & \multicolumn{2}{c|}{ Bacharelado } & Total \\
\cline { 2 - 6 } & $\mathbf{n}$ & $\mathbf{9}$ & $\mathbf{n}$ & $\mathbf{\%}$ & $\mathbf{n}$ \\
\hline Goiás & 7 & 11,2 & 5 & 7,9 & 12 \\
Distrito Federal & 7 & 11,2 & 18 & 28,6 & 25 \\
Mato Grosso & 8 & 12,7 & 7 & 11,2 & 15 \\
Mato Grosso do Sul & 6 & 9,5 & 5 & 7,9 & 11 \\
\hline \multicolumn{1}{c}{ Total } & $\mathbf{2 8}$ & $\mathbf{4 4 , 6 \%}$ & $\mathbf{3 5}$ & $\mathbf{5 5 , 6 \%}$ & $\mathbf{6 3}$ \\
\hline
\end{tabular}

Similar aos achados encontrados nos cursos de Nutrição pôde-se constatar que para a Educação Física a maioria dos cursos $(81,0 \%)$ também é ofertada por instituições privadas quando comparada a oferta por instituições públicas $(15,9 \%)$ e especiais $(3,2 \%)$.

Em relação ao perfil da disciplina avaliada, observou-se que para $100 \%$ dos cursos de $\mathrm{Nu}$ trição ela é considerada de natureza obrigatória, sendo ofertada entre o $4^{\circ}$ (quarto) e o $8^{\circ}$ (oitavo) período, predominando o $5^{\circ}$ (quinto) período, com $39,1 \%$ dos cursos.

Interessantemente, pôde-se notar uma ampla variabilidade na carga horária total da disciplina entre os cursos dos diferentes estados da Região Centro-Oeste e DF: mínima de 30 e máxima de 80 horas, com predomínio das cargas horárias de $30(n=4)$ e 60 horas $(n=4)$. Por estado e DF, a maior carga horária total para o curso de Nutrição foi atribuída ao Mato Grosso e a menor ao DF, conforme demonstrado abaixo (Tabela 3).

Ao avaliar a carga horária da disciplina destinada à parte teórica e prática no curso de Nutrição, verificou-se que as cargas horárias teóricas mais elevadas estavam presentes nos estados que não ofertavam a prática da disciplina (MT e MS). Contrariamente, o estado de Goiás apresentou a maior carga horária prática, embora seja o estado com menor carga horária teórica (Tabela 4).

Para a Educação Física, 95,2\% dos cursos oferecem a disciplina de modo obrigatório $(n=60)$ e os demais $(n=3)$ de forma optativa, sendo ofer-

Tabela 3. Carga horária total da disciplina de Nutrição em Esporte nos cursos de Graduação em Nutrição e Educação Física por estado e DF, na Região Centro-Oeste do Brasil.

\begin{tabular}{l|c|c|c|c}
\hline \multirow{2}{*}{ Estados e DF* } & \multicolumn{2}{|c|}{$\begin{array}{c}\text { Nutrição }(\mathbf{n = 2 1 )} \\
\text { Meducação Física (n=63) }\end{array}$} \\
\cline { 2 - 5 } & $\begin{array}{c}\text { Carga Horária Total } \\
\text { (horas) }\end{array}$ & \multicolumn{2}{c}{$\begin{array}{c}\text { Carga Horária Total } \\
\text { (horas) }\end{array}$} \\
\cline { 2 - 5 } & Média & DP* & Média & DP* \\
\hline Goiás & 49,2 & 17,1 & 61,6 & 7,8 \\
Distrito Federal* & 47,5 & 17,1 & 54,3 & 18,0 \\
Mato Grosso & 66,7 & 11,5 & 46,6 & 11,5 \\
Mato Grosso do Sul & 61,7 & 21,9 & 73,7 & 16,0 \\
\hline Geral & 56,3 & 16,9 & 59,0 & 13,3 \\
\hline
\end{tabular}


Tabela 4. Carga horária teórica e prática da disciplina de Nutrição em Esporte nos cursos de Graduação em Nutrição e Educação Física por estado e DF, na Região Centro-Oeste do Brasil.

\begin{tabular}{|c|c|c|c|c|c|c|c|c|}
\hline \multirow{3}{*}{ Estado e DF* } & \multicolumn{4}{|c|}{ Nutrição ( $n=21)$} & \multicolumn{4}{|c|}{ Educação Física (n=63) } \\
\hline & \multicolumn{2}{|c|}{$\mathrm{CH}^{* *}$ teórica } & \multicolumn{2}{|c|}{$\mathrm{CH}^{* *}$ prática } & \multicolumn{2}{|c|}{$\mathrm{CH}^{* *}$ teórica } & \multicolumn{2}{|c|}{$\mathrm{CH}^{\star *}$ prática } \\
\hline & Média & $\mathrm{DP}^{\star * * *}$ & Média & $\mathrm{DP}^{* * *}$ & Média & $\mathrm{DP}^{* \star * *}$ & Média & $D^{* * * *}$ \\
\hline Goiás & 42,1 & 15,1 & 7,4 & 13,1 & 58,2 & 14,3 & 3,3 & 7,8 \\
\hline $\mathrm{DF}^{*}$ & 44,4 & 16,7 & 4,3 & 11,3 & 51,4 & 19,1 & 3,0 & 8,7 \\
\hline Mato Grosso & 66,7 & 11,5 & 0 & 0 & 43,4 & 12,1 & 1,9 & 5,1 \\
\hline Mato Grosso do Sul & 61,7 & 21,9 & 0 & 0 & 73,7 & 16,0 & 0 & 0 \\
\hline Geral & 53,7 & 4,3 & 2,9 & 7,1 & 56,7 & 2,9 & 2,0 & 3,9 \\
\hline
\end{tabular}

tada entre $\circ 1^{\circ}$ (primeiro) e o $8^{\circ}$ (oitavo) período, prevalecendo o $4^{\circ}$ período com $23,8 \%$ dos cursos e com a seguinte distribuição: bacharelado: 27 obrigatórias e 01 optativa $(n=28)$ e licenciatura: 33 obrigatórias e 02 optativas $(n=35)$.

Em relação à carga horária total da disciplina avaliada, essa também apresentou grande variabilidade, com variação mínima de 30 e máxima de 90 horas, predominando 40 horas $(n=15)$. Diferentemente da Nutrição, a maior e a menor carga horária total foi atribuída, respectivamente, ao Mato Grosso do Sul e Mato Grosso (Tabela 3).

Ao avaliar a carga horária da disciplina destinada às aulas teóricas e práticas dos cursos de Educação Física, o Mato Grosso do Sul foi o estado que apresentou a maior carga horária teórica, não sendo ofertada parte prática nesse estado, ao passo que Goiás permaneceu com a maior carga horária de aulas práticas (Tabela 4).

Após comparar a diferença de carga horária da disciplina avaliada entre os cursos de Licenciatura e Bacharelado em Educação Física, notou-se que a disciplina tem maior carga horária total e teórica no bacharelado, ao contrário da licenciatura, que apresenta a maior carga horária prática (Tabela 5).

Tabela 5. Comparação da carga horária total, teórica e prática da disciplina de Nutrição em Esporte nos cursos de Graduação em Educação Física (licenciatura e bacharelado) por Estado e DF, na Região Centro-Oeste do Brasil.

\begin{tabular}{|c|c|c|c|c|c|c|c|c|c|c|c|c|}
\hline \multirow{3}{*}{$\begin{array}{l}\text { Estados } \\
\text { e DF* }\end{array}$} & \multicolumn{6}{|c|}{ Licenciatura } & \multicolumn{6}{|c|}{ Bacharelado } \\
\hline & \multicolumn{2}{|c|}{$\mathrm{CH}^{\#}$ TOTAL } & \multicolumn{2}{|c|}{$\mathrm{CHT}^{\# \#}$} & \multicolumn{2}{|c|}{$\mathrm{CHP}^{\# \# \#}$} & \multicolumn{2}{|c|}{$\mathrm{CH}^{\#}$ TOTAL } & \multicolumn{2}{|c|}{$\mathrm{CHT}^{\# \#}$} & \multicolumn{2}{|c|}{$\mathrm{CHP}^{\# \# \#}$} \\
\hline & Média & $\mathrm{DP}^{+}$ & Média & $\mathrm{DP}^{+}$ & Média & $\mathrm{DP}^{+}$ & Média & $\mathrm{DP}^{+}$ & Média & $\mathrm{DP}^{+}$ & Média & $\mathrm{DP}^{+}$ \\
\hline $\mathrm{DF}^{*}$ & 49,7 & 16,5 & 44,6 & 13,8 & 5,1 & 13,6 & 56,1 & 18,7 & 54,0 & 20,5 & 2,1 & 6,1 \\
\hline $\mathrm{GO}^{* *}$ & 59,3 & 9,4 & 53,6 & 17,5 & 5,7 & 9,8 & 64,8 & 3,3 & 64,8 & 3,3 & 0 & 0 \\
\hline $\mathrm{MT}^{* \star *}$ & 42,9 & 10,9 & 39,4 & 12,1 & 3,5 & 6,8 & 48,0 & 11,3 & 48,0 & 11,3 & 0 & 0 \\
\hline$M S^{* \star \star *}$ & 63,3 & 19,7 & 63,3 & 19,7 & 0 & 0 & 82,0 & 4,5 & 82,0 & 4,5 & 0 & 0 \\
\hline TOTAL & 53,8 & 14,1 & 50,2 & 15,8 & 3,6 & 7,5 & 62,7 & 9,4 & 62,2 & 9,9 & 0,5 & 1,5 \\
\hline
\end{tabular}

DF*: Distrito Federal; GO**: Goiás, MT ${ }^{\star * *}, \mathrm{MS}^{\star * \star *}$ :Mato Grosso do Sul; ${ }^{\#} \mathrm{CH}$ Total: Carga Horária Total (horas); ${ }^{\#} \mathrm{CHT}$ : Carga Horária Teórica (horas); ${ }^{\# \#} \mathrm{CHP}$ : Carga Horária Prática (horas); DP+: Desvio Padrão. 


\section{DISCUSSÃO}

Dentre os principais achados do presente estudo, do total de 21 cursos de graduação em Nutrição e 63 em Educação Física que foram analisados na Região Centro-Oeste do Brasil e DF pôde-se observar um elevado percentual de cursos de Nutrição (100\%) e Educação Física $(95,23 \%)$ que ofertam a disciplina de Nutrição em Esporte em suas matrizes curriculares de forma obrigatória. Uma pesquisa conduzida pelo CRN $2^{\text {a }}$ Região demonstrou que das 23 IES desse estado que oferecem o curso de Nutrição, $87 \%$ delas contém alguma disciplina ligada ao Esporte em sua matriz curricular, sendo que desse total, $57 \%$ desenvolvem algum projeto prático na área ${ }^{8}$.

Diferentemente, um outro estudo apontou que dos 762 cursos de graduação em Educação Física existentes em todo o país no ano de 2008, a disciplina de Nutrição em Esporte estava presente em apenas $20,47 \%$ dos cursos. Ao avaliarem as diferentes regiões, os autores verificaram que na Região Centro-Oeste, $29,62 \%$ dos cursos oferecia a disciplina em questão, um percentual bastante inferior ao encontrado no presente estudo $(95,23 \%)$. Segundo os autores, os cursos de graduação em Educação Física contribuíam pouco com a abrangência, desenvolvimento e com o processo de consolidação da Nutrição Esportiva, propondo a alteração na organização da Matriz Curricular. Cabe ressaltar que os autores não especificaram se os cursos de Educação Física avaliados foram licenciatura, bacharelado ou ambos. Em adição, os diferentes estudos foram conduzidos em períodos diferentes (2008 versus 2016), o que possivelmente pode explicar as discrepâncias nos resultados encontrados, dado que novas IES e cursos de Educação Física provavelmente foram criados nesse intervalo de tempo ${ }^{9}$.

Na última década, tem ocorrido um crescimento acentuado no número e temas de publicações relacionados à nutrição esportiva, tornando essa uma área dinâmica para a ciência e a prática e que vem continuamente se expandindo ${ }^{10}$. Logo, entende-se a inclusão da disciplina de Nutrição em Esporte como um reflexo consciente das IES em proporcionar uma formação profissional mais abrangente aos acadêmicos de Nutrição e Educação Física, oportunizando maior conhecimento e capacitação dos mesmos nessa área de atuação profissional.

A Resolução do Conselho Federal de Nutricionistas (CFN) (Resolução CFN n. 600, de 25 de fevereiro de 2018) define a Nutrição em Esportes e Exercício como uma das áreas de atuação do nutricionista e que compete a este profissional a execução de atividades relacionadas à alimentação e à nutrição em academias, clubes esportivos e similares ${ }^{6}$. O Conselho Federal de Educação Física (CONFEF) refere que cabe exclusivamente ao educador físico planejar, prescrever, supervisionar, orientar e dirigir programas de todas as atividades físicas, desportivas e similares, sendo ainda de sua competência prestar serviços que favoreçam o desenvolvimento da educação e da saúde, contribuindo para a capacitação e condicionamento fisiocorporal dos seus beneficiários, visando o bem-estar e a qualidade de vida ${ }^{11}$.

Por conseguinte, percebe-se que os dois profissionais se utilizam da nutrição esportiva por diferentes meios, para prestar atendimento a indivíduos praticantes de exercício físico. Embora não seja da competência do profissional de educação física a prescrição dietética, ele é considerado um importante veículo para a disseminação de informações, especialmente pela sua maior proximidade diária com atletas, frequentadores de academia e indivíduos fisicamente ativos em geral, bem como na educação escolar. Assim, torna-se relevante a abordagem de conteúdos de nutrição para a formação de profissionais minimamente instruídos nesta área de conhecimento ${ }^{12}$.

Em relação à carga horária total da disciplina avaliada, notou-se uma ampla variabilidade entre os cursos das diferentes IES. No entanto, como na presente investigação não houve uma avaliação qualitativa dos conteúdos e ementas, não foi possível inferir o impacto dessa diferença de carga horária na qualidade da disciplina. Contudo, os achados da presente investigação são de suma importância, pois demonstram a necessidade de futuros estudos avaliarem os conteúdos de nu- 
trição no esporte que são trabalhados nos cursos de Nutrição e Educação Física, a fim de proporcionarem um melhor entendimento sobre a qualidade em que tal disciplina é ofertada. Utilizar-se de estudos dessa natureza, conduzidos com as diferentes disciplinas existentes pode contribuir no processo de reavaliação e reestruturação dos currículos dos respectivos cursos de graduação, aprimorando, desta forma, a formação profissional ${ }^{13}$.

Adicionalmente, destaca-se a fragilidade da atuação integrada entre os estudantes da Nutrição e dos demais cursos da área da saúde, a exemplo da Educação Física. Somente na disciplina de estágio obrigatório há uma aproximação multiprofissional, no entanto restrita às atividades pontuais ${ }^{14}$.

Um achado interessante foi que em alguns estados existe cursos de graduação em Nutrição e Educação Física que não destinam nenhuma carga horária prática para a realização da disciplina em estudo. Esses resultados contrapõem o que as Diretrizes Curriculares Nacionais para o curso de Graduação em Nutrição (Resolução CNE/CES $N^{0}$. 5/2001) retrata em seu artigo 14, inciso II, ao elucidar a importância de atividades teóricas e práticas presentes desde o início do curso, permeando toda a formação do Nutricionista, de forma integrada e interdisciplinar. Também, entende-se que a participação do graduando em atividades práticas estimula um ensino mais crítico, reflexivo e criativo, atendendo, desta forma, a proposta das Diretrizes supracitada ${ }^{4}$. De modo similar, as Diretrizes Curriculares Nacionais para os cursos de Graduação em Educação Física (Resolução CNE/CES $N^{\circ}$. 7/2004) ressalta que a formação

\section{REFERÊNCIAS}

1. Nogueira JA, Da Costa TH. Nutrient intake and eating habits on triathletes on a Brazilian diet. Int J Sport Nutr Exerc Metab. 2004; 14(6): 684-97.

2. Torres-Mc Gehee T, Piritchett K, Zippel D, Minton D, Cellamare A, Sibilia M. Sports nutrition knowledge among collegiate athletes, coaches, athletic trainers, and strength and conditioning specialists. J Athl Train. 2012; 47(2): 205-211. do graduado em Educação Física deve assegurar a indissociabilidade teoria-prática e que a prática como componente curricular deverá ser contemplada no projeto pedagógico, sendo vivenciada em diferentes contextos de aplicação acadêmico-profissional, desde o início do curso ${ }^{5}$. A partir da observação de que alguns estados não ofertam carga horária prática para a disciplina avaliada, torna-se imprescindível a realização de estudos posteriores que investiguem a influência desse aspecto na qualidade do ensino-aprendizagem desta disciplina.

Portanto, torna-se necessário especificar se as diferenças nos perfis de oferta impactam na qualidade do processo ensino-aprendizagem, ou seja, entender se aquelas IES que contém maiores cargas horárias teóricas têm o processo de ensino-aprendizagem tão completa quanto àquelas que têm uma carga horária teórica menor, mas com elevadas cargas horárias práticas. Será que dentro desta carga horária teórica não caberia uma reformulação e, assim, inclusão de aulas práticas? Visto que as próprias DNC dos cursos de Nutrição e Educação Física enfatizam a importância e insociabilidade da teoria-prática em diferentes contextos e do início ao final do curso.

\section{CONCLUSÃO}

Os resultados mostram que a maioria dos cursos de Nutrição e Educação Física da Região Centro-Oeste do Brasil e DF possuem a disciplina de Nutrição relacionada ao Esporte com natureza obrigatória. Também, pôde-se observar uma similaridade no perfil de oferta entre os cursos de Nutrição e Educação Física para a maioria dos aspectos avaliados. 
5. Resolução CNE/CES N 7, de 31 de março de 2004. Diretrizes Curriculares Nacionais para os cursos de graduação em Educação Física, em nível superior de graduação plena. Brasília, DF: Ministério da Educação. Diário Oficial da União, Brasília, 5 de abril de 2004.

6. Resolução CFN No 600 , de 25 de fevereiro de 2018 . Conselho Federal de Nutricionistas. Dispõe sobre a definição das áreas de atuação do nutricionista e suas atribuições, estabelece parâmetros numéricos de referência, por área de atuação, e dá outras providências. Brasília, DF; 2018.

7. The IHRSA Global Report. Global Health Club Industry Posts Growth. International Health, Racquet \& Sports club Association. 2016: 21-26.

8. Conselho Regional de Nutricionistas. $2^{a}$ Região. Nutrição na Prática Esportiva - Nutrição em Esportes. Rev CRN 2. $2012 ; 28(1): 4-15$

9. Navarro AC, Navarro FN, Navarro F, Mercadante LA. Quantificação da disciplina curricular de nutrição esportiva e da carga horária nos cursos de graduação em Educação Física no Brasil. In: $61^{\text {a }}$ Reunião Anual da SBPC Amazônia e Cultura; 2009; Manaus, Brasil. Manaus: $61^{\text {a }}$ Reunião Anual da SBPC; 2009

10. American College of Sports Medicine (ACSM). Nutrition and Athletic Performance. Med Sci Sports Exerc. 2016; 48(3): 543-568.
11. Estatuto do Conselho Federal de Educação Física de 13 de dezembro de 2010. Conselho Federal de Educação Física (CONFEF). Rio de Janeiro, RJ: 2010. Diário Oficial da União, de 13 de dezembro de 2010.

12. Lollo PCB, Tavares MCGC, Montagner PC. Educação Física e Nutrição. Revista digital efdeportes.com. 2004; 10(79): 1.

13. Rios MTCA, Bruin MCB, Santos PL. Ensino Superior: a Psicologia na Formação do Nutricionista. RBCS. 2017; 21(1): 77-86.

14. Neves J, Sousa AA, Vasconcelos FAG. Formação em Nutrição em Saúde Coletiva na Universidade Federal de Santa Catarina: reflexões sobre o processo de ensino para fortalecer o Sistema Único de Saúde. Rev. Nutr. 2014; 27(6): 761-773.

\section{CORRESPONDÊNCIA}

Tatienne Neder Figueira da Costa

Av. NS 15 ALC NO 14, 109 Norte, Plano Diretor Norte

Cidade - Estado - País: Palmas, Tocantins, Brasil.

CEP: 77001-090

E-mail: tatienne_neder@outlook.com 